\title{
Tres cosas que se deberían saber antes de recibirse de diseñador
}

por Camilo Anabalón

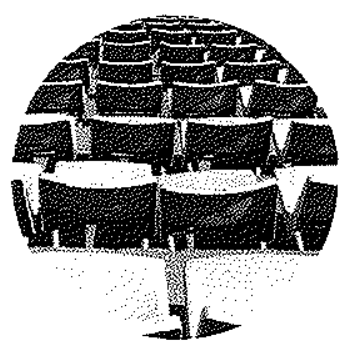

Palabras ctave:

Educacion, valores de diseño. procesos creativos
En Diseño, como en cualquier otra disciplina, se reciben junto con la titulación ciertas responsabilidades profesionales las cuales mayormente pueden ser clasificadas en tres áreas: la práctica, la disciplina en sí y la comunidad formada por sus pares.

Este ensayo trata acerca de tres conocimientos básicos que colindan con estas responsabilidades y que en el escenario actual del Diseño son herramientas fundamentales para la práctica profesional, así como para el desarrollo de la disciplina en sí, tanto para su desarrollo teórico como para su consolidación disciplinar. Estos son: El lenguaje de las máquinas, el lenguaje de la ciencia y el lenguaje de los procesos creativos.

La práctica del Diseño hoy en día está ligada profundamente a los medios digitales por lo que el producto del diseño depende en gran medida de la comprensión de las herramientas con los que este se produce, siendo la expresión más básica de esto los lenguajes de programación en los que se escriben los programas que hoy se utilizan para diseñar. Por otra parte, si el Diseño local pretende establecerse como voz válida dentro de la comunidad internacional ha de sistematizarse la experiencia, para lo cual es necesario marcar presencia dentro de los medios que dibujan las fronteras del campo disciplinar. Esto, publicando con oficio, persistencia y rigurosidad. Finalmente, la identidad del diseño frente a otras disciplinas así como su evolución interna depende en gran medida en cómo el diseñador hace lo que hace y la claridad que tiene al hacerlo, ya que a medida que entendemos lo que hacemos somos capaces de explicarlo, elaborarlo y mejorarlo. En este sentido la comprensión 
acerca de él o los procesos creativos -el mats obscuro de temas merodológicos en wiseños- - se ha clarificado bastante gracias al aporte tanto interno como el de otras disciplinas periféticas al Diseño, desmitificando el quehaser del diseñador y déndonos nuevas herramientas para enriquecer nuestra práctica.

\section{El lenguaje de las máquinas}

\section{De Las patas del tablero a las razanes de la pantalla}

El Diseño de hoy en día no es el mismo Diseño que aprendieron quienes hoy dirigen las escuelas de Diseño. Si bien el proceso de concepción e ideación ha carmbiado poco, el proceso de elaboración del diseño ha mutado sustantivamente debido a la incorporación de tecnologias digitales. El producto de diseño hoy está mediado poz softwares que nos permiten realizar nuestro trabajo no solo de manera rápida y precisa sino tämbién expresiva. El computador, hardwatre y software, se ha convertido en una herramienta funda. mental que nos permite explorar y expresar nucstras ideas, ¿cierto? Pues no del todo, aún con todo el avance tecnológico y el fantástico desarrollo de las interfaces sigue existiendo una brecha bastante importante. Los programas son, la gran mayoría de ellos, paquezes cerrados, cajas de herramientas de las cuales podemos exrraer el adminiculo que mấs se acerque a la función que necesitamos pero en la cual no bay espacio para la incorporación de nuevas herramientas. Puesto de mejor manera, la herramientas de estas cajas están hechas Con y De algo que no entendemos. Previamente a la revolución digital contábamos tambiétr con una caja de herramententas, entre ellas: lápices teglas, pinceles, pinturas, papeles, escuadras, tablero y un sinfin de orras más y menos especificas, pero que a diferencia de la caja digital tenta la capacidad casi infinita de seguir creciendo y especificáradose. A cada nueva necesidad o urgencia técnica o expresiva éramos - y creo lo segzimos siendo- capaces de claborar una nueva herramienta especifica para esa nueva tarea. Hoy en día si queremos agregar una herramienta a nuestra caja debemos descargarla o esperar la proxima actualización del sofuture, pero en ninguno de estos casos la herramienta sera la expresión ni la resolución de nuestra necesídad espectica. Siguiendo este mods1o la herramienta será siempre producto del trabajo de an tercero al cual probablemente nunca conoceremos. 
Esto genera dos problemáticas, por un lado lo que ya discutimos de la adecuación de la herramienta al trabajo que queremos realizar y segundo que nos genera uná dependencia económica intelectual para el desarrollo de nuestro trabajo. Por una parte económica ya que por lo general estas herramientas están protegidas por derechos de autor, patente y licencias las cuales deben ser costeadas si se quiere realizar trábajo con ellas. Y, por orra, intelectual ya que nuestras ideas han de ser mediadas o mas bien filtradas por herramientas que orros han disenado para tareas que ellos han dererminado relevantes. Estas herramicntas procedimentales procesaran nuestras ideas baipo un cierto progxama y generatan un producto con ellas por lo cual, si bien la idea es nuestra, el resulcado de esa idea es - en teoria - compartido por quien desarrolla y suministra el software usado para procesatla. La relevancia entonces del manejo de un lengazaje de programación va mas allá de simplementé entender de "Qué" y "Cóno" están hechas las herramientas que atilizamos para dar forma a nuestras ideas, si no que abarca la voralidad de la práctica desde sus procedimientos técnicos hasta su fundamento ético.

\section{Nuceos estenatios}

Sin embargo, el peso práctico de manejar este conocimiento tadica principalmente en los nuevos escenarios que se han abierto al $\mathrm{Di}$ seño a partir de la revolución digital. La generación de formas a través de medios digitales ya no se limita simplemente a la traducción de lo que antes se hacia en el tablero sino que nos permite nuevas aproximaciones a la geometría $-y$ por sxtension a los procesos productivos-en su dimensión más profunda. Lo hoy denominado "Diseño Generativo" (en adelante DG) ta permeado desde la pesquisa topológica hasta la fabricación del producto final. El De o Diseño en base a algoritmos le está parmitiendo al diseñador ampliar su vocabulario en tanto que da la posibilidad de jugat con la geometria de nuevas maneras aś como de dar ura dimensión física a estas a tavés de fabrieación directa. Las tecnologias digitales han hecho un puente que prescinde de la intervención humana y sus limitaciones (como lentieud, falta de precisión y necesidad de experiencia) entre la forma y su fabricación.

Por otra parte, estas tecnologias enzregan nuevas posibilidades para el protocipado de sistemas integrados, tan comunes en los objetos de hoy en día. El lenguaje de los productos de hoy considera no sólo el comportamiento de los usuarios sino el comportamiento de los objetos mismos, es decir, artefactos capaces de interactuar no sólo con el exromo físico del usuario sino que 
tambiér con el entorno cognitivo del sujeto. Attefactos inteligentes con comportamientos propios. Esto demanda la integración de sistcrnas electromecánicos con entornos digitales, más aún exige que los sistemas electromecánicos sean controlados por los entornos digitales. Control ejercido a travês de lenguajes de progamación. Es necesario entonces, si queremos desarrollar conscientemente un producto de estas caracteristicas, explorar y testear tanto su dimensión fisica como las funciones de su entorno digital y la calidad de la integración entre ambos sistemas. Esro se logra a través del prototipado, no necesariamente en alta resolución (Kelley zoon), de los componentes electromecánicos y de los comportamientos (entomo digital y su inftuencia sobre el sistema EM) del artefacto. Por lo tanto, se hace necesaria la integración de profesionales con experticia en el manejo de programación y controladores dentro del equipo de diseño y desarrollo o bien la incorporación de este conocimiento y habilidades en el cuerpo teórico-práctico del diseñador.

Otro escenario interesante que se ha desarrollado a partir de este cuerpo de conocimiento es el Disch́o de interacción, que busca generar entormos más sensibles y conscientes de sus interlocutores, a partir de la ldea de controlar mecanismos basados en la información recopilada por sensokes, dotando de inteligencia al arnbiente donde nos desenvolvernos. Encontramos ejemplos desde instalaciothes artisticas que simulan telas de arañas que atrapan al transeúnte ${ }^{x}$ hasta huces al interior de los autos gue responden con sutiles cambios de intensidad a la presencia o no de pasajeros. El Diseño de interacción requiere también del mancjo transversal de la cadena de control digital, desde al disenoo del comportamiento hasta la ejecución de la programación, fabricación y ensamblaje de las partes del mecarismo. Por esta razzón exige conocimiento y manejo tanto de algún lenguaje de programación como ambién de componentes electrónicos y electromecánicos además de agudeza de observación y capacidad de expresión.

\section{El lenguaje de la ciencia}

Primero que todo quisiera aclarar que con el lenguaje de la ciencia no me refiero a cómo la ciencia genera sus contenidos, el método cientifico, siro a cómo la ciencia comunica sus contenidos, es decir, cómo sus contenidos son presentados a la comunidad cientifica para su análisiss, validación e incorporación dentro del cierpo de conocimientos de determinado dominio. La ciencia busca explicaciones empiricas a los fenómenos de la naturaleza, para lo cual genera hipótesis que luego trata de comprobar por medio de experimentos

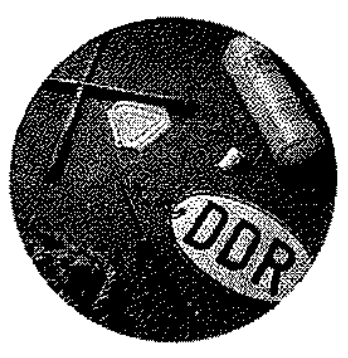




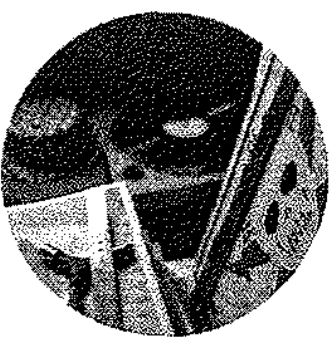

protocolarizados, es decir, reproducibles. Estos experimentos, sus datos, procesos y conclusiones, luego han de ser publicados bajo distíntos medios. Los medios no simplemente publican, sino que revisan la información entregada y dan validez a lo publicado, son los medios quients velan porque la novedad presentada al campo es información original y de valok. Todo este proceso tienc princhpalmente dos funciones: incorporar el nuevo conocimiento al campo de la disciplina, es decir, a quienes manejan su contenido $y$ practican en ella; y segundo, entregar nuevos argumentos a la permanente discusión á cómo comprendemos el mundo, como bien lo dice Pazel "La ciencia trata de discrepar sobre cosas qué podemos nuedir" (Patel zo06).

\section{De esqueleto a red}

Si bien la roción de Diseño como ciencia es una discusion nón bien abierta, a nivel incernacional se pueden ver señales de que ha estrucrura intema de diseño se está otganizando en base a la grilla de una disciplina científica. La apurición y consolidación de numerosos espacios de calidad para la discusión (más allá de revistas que muestran el estado dal aree y los últimos productos de la práctica), tales como Jouratsi, seminarios, congresos y encuentros, además de casas de publicación especializadas, da luces de cómo el cuerpo de conocimientos de nuestra disciplína se está estructurando alrededor de la generación de nuevos contenidos y de su discusión. Buen jemplo de exro es la lista elaborada por Designonhy con más de 60 conferencias sôlo para el segundo semestre de este año.

El Diseño ha cambiado su anatomáa disciplinar defando atrás un tsquel to teórico basado en escurlas, para evolucionar hacia un estado intermedio que dio paso a personajes específicos, gurtues, que actiaban como pilares de la reflexión en diseño (principalmente th su metodologha) llegando hoy en día a lin estado ke mayor disgregación más parecido a una red, donde el pensamiento, la crítica y La reflexión se articulaten una díscusión normaday con instancias definidas. Esto significa que el contenido y el peso de la discusión ya no responden a un programa sociopolítico o ideológico específico (como fue el caso de la Bauhaus y de la HfG Ulm) o a la sintesis de la experiencia putcica-reflexiva de un personaje, sino más bien a la consistencia y resonancia de los aroumentos presenrados. Es decir, la discusión no sólo se ha disgregado aumentando en variedad y ubiquidad, sino que ademas se ha vuelto mas amplia proporcionando espacios para distintas aproximaciones ya sea por linea teótca o por expresión culeural. 
Esta situación genera nuevas oportunidades para la prescnación de argumentos locales ya que, cumpliendo con los requerimizntos minimos de publicación, cstos han de ser igualmente balanceados en base a sa relevancia y originalidak, provengan de donde provengan. Prueba de esto es la presencia en prestigiosas publicaciones, de articulos provenientes desde lugares bastante lojanos a los tradicionales centros de desarrollo del Diserio, como es el caso de los numerosos artículos que hablan de Turquia (36) en Design $15 s t a s o$ el artículo acerca de la Escuela de Artes Aplicades de la Universidad de Chile por Ecúardo Castillo en la misme publicación.

\section{Campartir es construit}

El hảbito de escribir reportes, papers o artículos es parte importante en la eductión de las disciplinas cientificas, ya que es consecuencia directa de su nétodo; el proceso no termina con la realización del experimento sino con la presentación y discusión de sus resulcados (Collins 20ro). En Diseño, en canbio, el restaltado del proceso es, por logeneral, un producto, lo que también es consecuencia directa de di - los métodos aplicados al proceso de diseño. Si bien ha habido aproximaciones bastante interesantes de la aplicación del mátodo cientifico al proceso de disctio, la práctica difiere generabmente de esta aproximación dado que el Diserio es fundamentalmente experiencial (más que lógico), dado que más allá de redacir variables para su observación detallada, el diseño se nutre de experiencias previas y características personales para generar un producto por lo que anque establezcamos un proceso lincal y istratificado el resultado de este no será nexcsariamente el mismo (Parsons 2009)

Es precisamente esta caracteristica del Diseño lo que lo hace interesante para da teflexión sobre st quebacer principalmente por dos cosas: primero, porque la variedad de aproximaciones es tan nicha como el rámero de puacticantes y la discusión de estas distintas aproximaciones ha de generar un cuerpo de conocimiento comparado de gran utilidad para la práceica de la disciplina; y segundo, porque cn base a esto, el Diseño puede ser tomado como expresión de quienes lo desarrollan y del contexto en el cual lo hacen, ex decir, una expresión cultutal, de la cual pueden apatecer luces sobre la identidad, y por tanto las competencias, ventajas y desafios del Diseño a nivel lock.

Escribix en ana disciplina como el Diseño no es simplemente reportar, es reflexionar. Yes sobre la refiexión, la cricica y la discusión que se construye la comprensión y de esta ha de emanat el discurso disciplinar on consistencia y atonomia. 


\section{El lenguaje del crear}

Como discutiamos en la sección anterior, una de las principales características del Diseño es que se ve influenciado por nuestras experiencias previas y rasgos de personalidad, es más se nutre de ellas. Esto sucede ya que el Diseño lidia con la resolución de problemas de manera heurística, esto es, soluciona problemas en los cuales el camino a la resolución suele no estar definido (como en el caso opuesto de la resolución algorítmica de problemas) (Amabile x996) incorporando en el proceso a toda su persona. Es por esta manera de resolver problemas que al Diseño se le denomina una disciplina "Creativa”. Pero, ¿En qué parte del proceso ocurre esto? ¿Podemos manejar esta variable o somos presas de ella?

El proceso de diseño ha sido innumerables veces esquematizado en términos de pasos, etapas, tareas y objetivos que de una u otra manera tratan de expresar en términos lineales un proceso que suele no serlo. Hoy en día ha surgido un consenso de que el proceso de diseño se articula en base a una batería de verbos que definen la meta y las fronteras de las distintas etapas operativas del proceso; de esta manera definir, investigar, idear, prototipar, seleccionar, implementar y aprender se organizan en base a los requerimientos del proyecto y al proceder específico del diseñador (Ambrose y Harris 2010). En todas estas etapas es necesaria cierta cuota de creatividad, pero en algunas de ellas ha de ser más relevante. En las etapas de Definir, Idear y Prototipar junto con su labor especifica llevan la función de concepción, es donde emergen los constructos que guiarán y sustentarán el resto del proyecto, es donde surgen las ideas. Tal es la dependencia del producto final del Diseño en la concepción de nuevas ideas que el proceso de diseño en sí ha sido llamado "...la versión profesionalizada del proceso creativo" (Wylant 2008).

\section{Cómo fabricamos nuestras ideas}

El proceso de hace emerger ideas, o proceso creativo, ha sido tradicionalmente comprendido y estudiado en base a cinco etapas: Preparación, Incubación, Insight $t^{+}$, Evaluación y Elaboración (Csikszentmihalyi 1996). Cada persona que alguna vez haya tenido que corregir en último momento un proyecto, un escrito, un diseño, etc. Porque vio una mejor manera de expresar la idea o quizás vio una mejor idea en sí, sabe que este es un proceso no lineal en donde las etapas se superponen, cambian de orden y las súbitas luces de comprensión - que aquí llamamos Insight-surgen sin respetar estructuras ni horarios. Sin embargo es posible -e importante- 
caracterizar estas etapas separadamente para saber cómo manejarlas y navegar a través de ellas.

El generar nuevas ideas es una función del pensamiento y como acertadamente declara Humberto Maturana, nosotros no pensamos en el vacío. La primera etapa del proceso creativo, la Preparación, se trata esencialmente de esto, de construir un cúmulo de ideas sobre las cuales trabajar. La información contenida en estas ideas será la materia prima para la generación de otras nuevas.

En la segunda etapa, la Incubación es donde "trabajamos" estas ideas. Esto significa que tomamos esos paquetes de información que llamamos ideas, los desarmamos y utilizamos la información para construir nuevos paquetes, cuando uno de estos es identificado como una idea capaz de dar una solución coherente y novedosa al problema lo llamamos Insight. El Insight es tanto una etapa en sí como una parte de la incubación. Esto dado que generalmente el proceso de Incubación es llevado a cabo bajo el umbral de la conciencia, por lo que los mecanismos que hacen emerger los Insights, resultado visible a la consciencia del proceso de Incubación, no son del todo claros. Y por otra parte, la etapa del Insight puede ser trabajada conscientemente en base a las herramientas del pensamiento lateral (deBono 1970). Aún así trayendo el Insight por sobre el umbral de la conciencia, el complejo proceso de Incubación permanece esencialmente inconsciente integrando no sólo las ideas provenientes de las etapas previas sino que también incluyendo en el proceso nuestros rasgos personales y tensiones internas. En este sentido no es mucho lo que podemos intervenix en la parte inconsciente del proceso de Incubación, lo que sí podemos es hacer emerger sus contenidos hasta tenerlos al alcance de la conciencia. Esto podemos lograrlo "escuchando" nuestro subconsciente por medio de la fantasía y de la utilización de medios expresivos como puente entre lo inconsciente y lo consciente' (Jung $\times 916 / 58$ ). Es principalmente en estas etapas del proceso donde la concepción es llevada a cabo, es de aquí donde surgen las semillas del producto del Diseño, es por tanto fundamental reconocer estas etapas y educarnos en cómo hacer de ellas una herramienta fiable y por sobre todo consistente.

La cuarta etapa de proceso creativo es la Evaluación donde juzgamos a la nueva idea por su ajuste con el contexto para la cual fue creada. La emergencia de ideas suele venir acompañada de entusiasmo, energía y motivación por llevar a cabo la idea, pero hemos de ser cautelosos

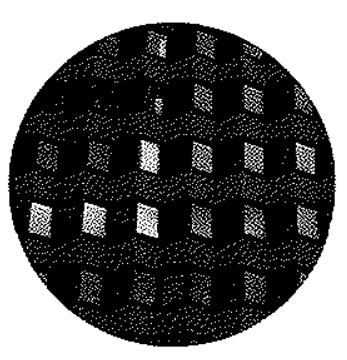
en darles peso correcto. No porque sea nueva significa que sea buena.

La etapa final del proceso de emergencia de ideas es la Elaboración, instancia en la que se buscan expresiones y variaciones para 
las nuevas ideas. Es aquí donde se realiza el penitente trabajo de darles la forma, consistencia y detalle necesario para que funcione dentro del contexto en el que se proyectó en un principio.

\section{Hacer mejor o hacer nuevo}

El cómogeneramos ideas resulta claramente relevante en las etapas de ideación y prototipado del proceso de diseño, donde lidiamos con un marco de acción más o menos claro y con problemáticas definidas. Pero lo que no resulta tan claro es que el rol más importante lo juega en la definición del problema. Gran parte del trabajo creativo radica en el planteamiento del problema, en cómo definimos la situación que queremos resolver o incluso en ver que hay una solución que necesita ser resuelta. Esto bajo la premisa que el nivel de novedad de resolver algo que ya ha sido resuelto anteriormente pero de manera discinta, no será nunca igual a resolver un problema que no se ha resuelto nunca antes. En esco radica la diferencia entre lo hoy llamado "innovación incremental" $\mathrm{e}$ "innovación disruptiva". Definir un problema es principalmente una tarea lingüística dado que la comprensión depende en gran medida en la manera en cómo articulemos la oración. De cómo comprendamos el problema dependerá cómo resolvemos el problema. La comprensión del proceso creativo es fundamental en la definición del problema porque definir es en sí un problema que no tiene una solución específica (al menos en temas de ciseño). Definir requiere heurística, no algorítmica, por lo tanto ha de ser resuelto de manera creativa.

\section{Conclusión}

El conocimiento y la solvencia de estos tres lenguajes son fundamentales en la educación del Diseño hoy en día porque son cada vez más importantes en la práctica y porque además son factores fundamentales para consistencia tanto interna y local de la disciplina como frente a sus vecinas periféricas. Es por eso que la formación de diseñadores debe apuntar, además de generar diseñadores para diseñar, a educar diseñadores para el Diseño. Profesionales con una visión y una preocupación no sólo de lo que pasa en el campo laboral sino que además en su campo disciplinar y ser conscientes de que su futuro laboral depende no solamente de la práctica, sino además en parte de la robustez de la disciplina en la que ejercen, la cual depende solamente de ellos. 


\section{Notas}

1. http//Www.Sagmeister.com/taxonomy/term/32 $\frac{4}{\pi} /$ node/216

2. Uso aqui la palabra inglesa para diferenciar lo que en español sería una revista normal de una revista de publicación cientifica.

3. http:/hww designophy.com/calendar/conferences.pho

4. Uso palabra inglesa para alejarme del sentido metafísico que pueden tener sus traducciones españolas "revelación" o "iluminación".

5. A esto el Psicoanalista C.G. Jung lo lamó ei método de la lmaginación Activa. El hacía a sus pacientes pintar, esculpir, escribir o incluso danzar y a partir de su interpretación lograba extraer contenidos del subconsciente.

6. Innovación disfuptiva o radical como la define Gold es "la creación ỏe cosas que generan nuevas categorias de nuevas cosas" (Gold 2007), por ejemplo, si un nevvo modelo depotivo de un CD player fue una innovación incremental, la introduccion oel MP3 fue una innovación radical.

\section{Bibliografia}

Amabilc. Teresa. "Crearivity in context." In Creativity in context. by Teresa Amabile. 1996.

Ambrosc, Gavin, and Paul Harris. Design thinking. lausanne: AVA Publishing SA. 2010.

Cascy Reas, Ben Fry. "Processing, a programing handbook for visual designers and artist." In Processing, by Ben Fry Casey Reas, r-5. Cambridge: mit press, 200\%.

Castillo, Eduardo. "The School of Applied Arts. University of Chile (1928-1968)". Design lssues 25. no. 2 (2009):75-93

Collins. Hilary. "Creative research, the theory and practice of rescarch for crearive industries". In creative rescarch, the theory and practicc of rescarch for crearive industrics, by Hilary Collins, 12. Lausanne: ava Publishing SA. 2010.

Csikszentmihalyi, Mihaly. "Creativity flow and the psychology of discovery and invention." In Crearivity. flow and the psychology of discovery and invention, by Mihaly Csikszentmihalyi. 79-80. 1996.

dcBono. Edward, Lateral thinking, creatirity secp by step. New York: Harper Perennial, 1970.

Gold. Rich. "The Plenituke." In The Plenitude. by Rich Gold, 5-6. cambridge: mit press, 200\%,

Jung. Carl Gustav. "Jung on active imagination." Chap, 2 in Jung on active imagination, by Carl Gustav Jung, partij2. 19:6/58.

Kelley. Tom. "The Arr of Innovation." In The Arr of Innovation, by Tom Kellcy, 101-118. London: Harper Collins Business. 2001.

Parsons, Tim. "Thinking: Objccts, contcmporary approaches to product design". In Thinking: Objects, contemporary approaches to produce design, by Tim Parsons, 155-159. Lausanne: AVA Publishing SA, 2009.

Patcl, Aniruddh. "Music and the Mind". hatp://youtubc/ZgKFcuzGEns (accessed o6 13.2011).

Wylant. Barry. "Design Thinking and the Experience of lnnovation". Design Issues 24. 1no. 2 (2008): ;-14. 